\title{
Sub-micron Magnetic Patterns and Local Variations of Adhesion Force Induced in Non-Ferromagnetic Amorphous Steel by Femtosecond Pulsed Laser Irradiation
}

Huiyan Zhang ${ }^{\mathrm{a}}$, Yuping Feng ${ }^{\mathrm{a}}$, Daniel Nieto ${ }^{\mathrm{b}}$, Eva García-Lecina $^{\mathrm{c}}$, Clare Mcdaniel $^{\mathrm{d}}$, Jordi Díaz-Marcos ${ }^{\mathrm{e}}$, María Teresa Flores-Arias ${ }^{\mathrm{b}}$, Gerard M O'connor ${ }^{\mathrm{d}}$, Maria Dolors Baró $^{\mathrm{a}}$, Eva Pellicer ${ }^{\mathrm{a}, *}$ and Jordi Sort ${ }^{\mathrm{f}, \S}$

åDepartament de Física, Universitat Autònoma de Barcelona, E08193 Bellaterra, Spain.

${ }^{\mathrm{b}}$ Microoptics and GRIN Optics Group, Applied Physics Department, University of Santiago de Compostela, E15782 Santiago de Compostela, Spain.

'Unidad de Superficies Metálicas, IK4-CIDETEC, E20009 Donostia-San Sebastián (Gipuzkoa), Spain.

${ }^{\mathrm{d}}$ National Centre for Laser Applications, School of Physics, National University of Ireland, Galway, Ireland.

${ }^{e}$ Unitat de Tècniques Nanomètriques, Centres Científics i Tecnològics, Universitat de Barcelona, E08028 Barcelona, Spain.

Institució Catalana de Recerca i Estudis Avançats (ICREA) and Departament de Física, Universitat Autònoma de Barcelona, E08193 Bellaterra, Spain.

To whom correspondence should be addressed: *eva.pellicer@uab.cat; §iordi.sort@uab.cat

Keywords: Femtosecond pulsed laser irradiation, amorphous steel, magnetic patterning, surface ripples, adhesion force, wettability

This is the author's version of a work that was accepted for publication in Applied surface science (Ed. Elsevier). Changes resulting from the publishing process, such as peer review, editing, corrections, structural formatting, and other quality control mechanisms may not be reflected in this document. Changes may have been made to this work since it was submitted for publication. A definitive version was subsequently published in Zhang, $\mathrm{H}$. et al. "Submicron magnetic patterns and local variations of adhesion force induced in non-ferromagnetic amorphous steel by femtosecond pulsed laser irradiation" in Applied surface science, vol. 371 (May 2016), p. 399-406. DOI 10.1016/j.apsusc.2016.03.011 


\begin{abstract}
Periodic ripple and nanoripple patterns are formed at the surface of amorphous steel after femtosecond pulsed laser irradiation (FSPLI). Formation of such ripples is accompanied with the emergence of a surface ferromagnetic behavior which is not initially present in the non-irradiated amorphous steel. The occurrence of ferromagnetic properties is associated with the laser-induced devitrification of the glassy structure to form ferromagnetic $\left(\alpha-\mathrm{Fe}\right.$ and $\left.\mathrm{Fe}_{3} \mathrm{C}\right)$ and ferrimagnetic $\left[(\mathrm{Fe}, \mathrm{Mn})_{3} \mathrm{O}_{4}\right.$ and $\left.\mathrm{Fe}_{2} \mathrm{CrO}_{4}\right]$ phases located in the ripples. The generation of magnetic structures by FSPLI turns out to be one of the fastest ways to induce magnetic patterning without the need of any shadow mask. Furthermore, local variations of the adhesion force, wettability and nanomechanical properties are also observed and compared to those of the as-cast amorphous alloy. These effects are of interest for applications (e.g., biological, magnetic recording, etc.) where both ferromagnetism and tribological/adhesion properties act synergistically to optimize material performance.
\end{abstract}

\title{
1. Introduction
}

The unprecedented technological progress in diverse areas of nanoscience and nanotechnology is raising an increasing demand for new types of materials, novel lithographic procedures and new techniques to assemble micro-/nanocomponents into complex systems, such as micro-/nanodevices, lab-on-a-chip or miniaturized robotic platforms. To a large extent, coping with the current technological challenges relies on the implementation of innovative methods to fabricate large areas of patterned structures with sub-micrometer scale precision in a rapid, inexpensive and industrially scalable manner.

High-resolution magnetic patterning is crucial in applications like magnetic encoding, magnetic sensors and actuators, wirelessly actuated magnetic microrobots, spin-electronics or high-density magnetic recording media. In all these applications, not only the magnetic properties are important but other aspects, such as surface adhesion, nanomechanical behavior or hydrophobicity need to be precisely controlled in order to attain optimized material performance. The tribology behaviour of patterned magnetic materials and the quantification of surface adhesion forces, for example, are of 
paramount importance in high-density recording media, where the write/read heads fly only a few $\mathrm{nm}$ from the surface of the recorded information [1]. Adhesion is a crucial parameter in magnetic systems for biological and mechatronic applications [2,3], in some bioinspired actuator devices [4] or in magnetic wall-climbing devices [5]. Surface roughness plays also a crucial role on the magneto-electrical properties of a variety of thin films $[6,7]$.

In many of these cases it is desirable that the magnetic behavior of the patterned structures is not affected by the presence of magnetic stray fields stemming from neighboring magnetic materials or underlying magnetic layers. For this reason, sophisticated lithographic procedures, that are usually rather time-consuming (i.e., consisting of multiple steps) and rather costly (i.e., requiring clean room facilities), are utilized to fabricate arrays of ferromagnetic dots at the surface of non-ferromagnetic substrates [8].

In the last few years, some non-conventional approaches for the direct magnetic patterning of initially non-magnetic materials have been developed. These include, for example, nanoindentation and ion irradiation of atomically-ordered alloys, stainless steels or metallic glasses [9-13]. The large compressive stresses generated during nanoindentation are sufficient to induce atomic order-disorder transitions in $\mathrm{Fe}-\mathrm{Al}$ alloys, local nanocrystallization in metallic glasses or martensitic transformations in austenitic steels. In all these cases, the induced structural changes result in localized magnetic patterning at specific regions of the sample surface [9-11]. Ion irradiation through shadow masks is an alternative procedure to fabricate arrays of ferromagnetic structures (sometimes without any topological damage) in some of the aforementioned materials $[12,13]$.

Femtosecond pulsed laser irradiation (FSPLI) is a powerful technique to induce periodic topological patterning and concomitant ablation damage at the surface of certain metallic alloys, semiconductors, dielectrics and polymers [14-24]. Depending on the laser processing conditions (fluence and nominal number of pulses) the imprinted structures can change from low-spatial-frequency ripples or "classical ripples" with periodicity close to the laser light (perpendicular to the polarization of the incident laser beam) $[9,10]$ to high-spatial-frequency nanoripples with periodicity smaller than laser wavelength (either perpendicular or parallel to the polarization direction [17-19]. Even 
spikes [20], regular arrays of nanopores [21] or concentric rings [22] can be induced under certain circumstances. Several models have been put forward in the literature to account for the various types of ripple topologies. Examples include the interference between the laser beam and the surface scattered wave, the excitation of surface plasmon polaritons, and self-organization. Regarding interference, it is proposed that when a laser beam interacts with a material, surface defects cause the incoming incident laser beam to become partially scattered into a tangential wave, which propagates across the material surface. Laser-induced periodic surface structures are thought to appear because of interference of the laser beam scattered by multiple surface defects [23]. Separately, it has been reported that when the incident electromagnetic laser radiation couples with a plasmon charge oscillation, a modified electromagnetic field propagates in the area which has specific polarisation dependence, regardless of whether a material is metallic or dielectric in nature [24-26]. For metals, a specific dependence of the plasmon coupling has been reported depending on the grating period [27]. Furthermore, ripple formation has been compared to the formation of sand dunes present in a desert and to other structures spontaneously created following ion beam sputtering on a microscale [28]. These spontaneous processes include the melting and resolidification processes occurring at the surface due to the induced temperature gradients [29-31]. Additionally, second harmonic generation has been shown to play a role in the formation of nanoripples [32]. In spite of the very short duration of the pulses, phase transformations and other microstructural changes are sometimes observed during FSPLI, for example amorphization of Si [15], crystallization of chalcogenides [33] or crystallization of metallic glasses [31,34].

In addition to its fundamental interest, surface treatment using FSPLI is also appealing for practical applications, as a method to increase the corrosion resistance of steel [35], to induce superhydrophobicity in several alloys [36] or to improve the bending plasticity in certain types of metallic glasses [37]. Remarkably, most of previous works dealing with FSPLI have focused on the mechanisms responsible for topological surface corrugation or on the variation of macroscopic physico-chemical properties at the surface level treating the material as a whole [18,19,38-42]. Conversely, detailed mappings of physical properties at the micro-/nanoscale (i.e., at the "ripples" length-scales) have been mainly overlooked. In particular, the use of FSPLI as a method to induce magnetic patterning has not been demonstrated. 
In this work, we report on the utilization of FSPLI to induce magnetic patterning at the surface of $\mathrm{Fe}_{51} \mathrm{Mn}_{10} \mathrm{Cr}_{4} \mathrm{Mo}_{12} \mathrm{C}_{15} \mathrm{~B}_{6} \mathrm{Er}_{2}$ alloy. $\mathrm{Fe}(\mathrm{Co})-\mathrm{Cr}-\mathrm{Mo}-\mathrm{C}-\mathrm{B}-\mathrm{Ln}$ and $\mathrm{Fe}-\mathrm{Cr}-$ $\mathrm{Mn}-\mathrm{Mo}-\mathrm{C}-\mathrm{B}-\mathrm{Ln}$ (where $\mathrm{Ln} \equiv$ lanthanide element) alloys, with $\mathrm{Fe}(\mathrm{Co})$ content around 50 at.\%, have been intensively investigated during the last few years owing to their high glass forming ability, outstanding mechanical properties and high corrosion resistance $[43,44]$. For certain compositional ranges, the presence of small metalloid atoms (C,B) together with large refractory metals $(\mathrm{Cr}, \mathrm{Mo})$ promotes the formation of a glassy microstructure which does not exhibit long-range crystallographic order. In these alloys, $\mathrm{Mn}$ is known to play a crucial role in increasing the reduced glass transition temperature [45], while suppressing the ferromagnetic behavior at room temperature. These materials, often referred to as "non-ferromagnetic amorphous alloys", exhibit a yield stress that can be about three times larger than that of crystalline austenitic stainless steel. The alloy composition chosen in our study corresponds to the one that exhibits the largest glass forming ability among the $\mathrm{Fe}-\mathrm{Cr}-\mathrm{Mo}-\mathrm{C}-\mathrm{B}-\mathrm{Ln}$ alloy system [46]. A selfinduced mechanism leading to the formation of periodic microstructures has been observed during femtosecond pulsed laser ablation at the surface of this material. Besides the formation of low-spatial-frequency ferromagnetic ridges perpendicular to the polarization of the incident laser beam, high-spatial-frequency nanoripples (parallel to the laser polarization) are also formed between consecutive low-frequency ridges. The observed ferromagnetic behavior is ascribed to surface-crystallization of some ferromagnetic $\left(\alpha-\mathrm{Fe}, \mathrm{Fe}_{3} \mathrm{C}\right)$ or ferrimagnetic [like $(\mathrm{Fe}, \mathrm{Mn})_{3} \mathrm{O}_{4}$ or $\mathrm{Fe}_{2} \mathrm{CrO}_{4}$ ] phases during the FSPLI process. These results show a potentially new application of FSPLI, as a tool to magnetically pattern large areas $\left(\sim \mathrm{cm}^{2}\right)$ of a non-magnetic material in a very fast (few seconds) and inexpensive manner. Furthermore, a mapping of the local variation of nano-mechanical properties (adhesion force) at the FSPLI treated surface was obtained by atomic force microscopy (AFM) with Peak Force tapping mode.

\section{Experimental procedure}

The $\mathrm{Fe}_{51} \mathrm{Mn}_{10} \mathrm{Cr}_{4} \mathrm{Mo}_{12} \mathrm{C}_{15} \mathrm{~B}_{6} \mathrm{Er}_{2}$ alloy was prepared by arc-melting a mixture of the high purity elements (99.9 wt.\%) under a Ti-gettered Ar atmosphere. The master alloy was then injected into a copper mold to produce cylindrical rods with a diameter of 5 $\mathrm{mm}$. The rods were subsequently cut into discs with a thickness of $2 \mathrm{~mm}$ and polished using diamond paste. The FSPLI treatment was performed in air (ambient conditions) using s-Pulse HP (Amplitude Systemes) comprising a Diode Pumped Femtosecond 
laser based on Ytterbium crystal technology. The incident direction of the laser was orthogonal to the sample plane. The irradiation conditions were: applied fluence of 0.33 $\mathrm{J} / \mathrm{cm}^{2}$, laser spot of $20 \mu \mathrm{m}$, wavelength of $1030 \mathrm{~nm}$, repetition rate of $100 \mathrm{kHz}$, pulsewidth of $500 \mathrm{fs}$ and sample scan speed of $200 \mathrm{~mm} / \mathrm{s} ; 50$ passes of highly overlapped $(95 \%)$ pulses were used. Large areas (of $3 \times 3 \mathrm{~mm}^{2}$ ) as well as arrays of squares, circles and lines (with widths down to $20 \mu \mathrm{m}$ ) were prepared using the same irradiation conditions. Note that, contrary to other studies where the sample is irradiated $N$ times at each given location, in our case the sample was kept in motion during the surface treatment and a strong pulse overlapping was employed. This method is very effective in accelerating the processing times while the obtained results are similar to conventional pulsed laser treatments. For instance, an area of $1 \mathrm{~cm}^{2}$ can be processed in about only 10 seconds. Structural characterization of the as-cast and laser treated samples was carried out by x-ray diffraction (XRD) using $\mathrm{Cu} \mathrm{K}_{\alpha}$ radiation (Philips X'Pert diffractometer). The surface morphology was investigated by two separate AFMs, i.e., an Agilent 5500 and a Multimode 8 with Nanoscope electronics $V$ from Bruker. Both the surface roughness and adhesion were evaluated, the latter using the Peak Force tapping QNM mode from Bruker. Detailed observations of the ripples were performed by means of field-emission scanning electron microscopy (FESEM, Zeiss Merlin), coupled with energy-dispersive X-ray (EDX) for compositional analyses. Magnetization versus applied field curves were recorded at room temperature by means of a vibrating sample magnetometer (VSM) from Oxford Instruments. The generated ferromagnetic patterns were observed by magnetic force microscopy (MFM) at a lift height of $100 \mathrm{~nm}$ using a setup from Danish Microengineering and Si tips coated with CoCr alloy. Nanoindentation experiments were performed using a UMIS indenter from Fischer Cripps Laboratories, with a Berkovich-type pyramidal-shaped diamond tip, in load control mode, applying a maximum load of $10 \mathrm{mN}$. The hardness and reduced Young's modulus were calculated from the unloading indentation segments, using the method of Oliver and Pharr [47]. Finally, contact-angle measurements were performed using the sessile-drop technique $(5 \mathrm{wt} . \% \mathrm{NaCl}$ aqueous solution droplets with $1 \mu \mathrm{L}$ volume).

\section{Results and discussion}

Fig. 1 (a) shows some examples of the patterns that can be induced by FSPLI imaged by SEM. Honeycomb arrays of circular dots, digits and letters and networks of 
stripes can be drawn at the surface of the metallic glass in a very fast way (few seconds), with a precision determined by the laser spot (in this case $20 \mu \mathrm{m}$ ). The processing times are orders of magnitude faster than those involved in multi-step conventional patterning methods such as optical or electron beam lithography. The lateral resolution of the areas printed by FSPLI is comparable to the one attained with other direct patterning techniques, such as inkjet printing technology (typically of several tens of $\mu \mathrm{m}$ ), that still requires, in any case, of shadow masks [48].

Fig. 1 (b) reveals that surface corrugation occurs at the micrometer length scale inside each laser irradiated spot. Periodic arrays of ripples, oriented perpendicular to the laser polarization $(P)$, are formed spontaneously [Fig. 1 (c)]. The periodicity of these "classical ripples" is $770 \pm 28 \mathrm{~nm}$, hence slightly smaller than the wavelength of the laser, in agreement with other works from the literature [18,20,21,32]. The depth of the ridges is $238 \pm 9 \mathrm{~nm}$ [Fig. 2 (a) and (b)]. The magnified views of the classical ripples at the FSPLI treated surfaces, with and without vertically tilting the sample plane by $45^{\circ}$ with respect to the $x-y$ plane are shown in Fig. 3. Remarkably, abundant nanoripples are observed between consecutive ridges by the high-resolution SEM. The orientation of the nanoripples is perpendicular to the classical ripples (i.e., parallel to the polarization of the incident laser). The nanoripples exhibit average width of $\sim 100-300 \mathrm{~nm}$ [Fig. 3 (a)] and thickness of few tens of nm [Fig. 3 (b)]. The formation of nanoripples between classical ripples has been previously reported according to the surface plasmon interference model combined with grating-assisted coupling. The high-spatial-frequency nanoripples are formed as common walls between adjacent bursting bubbles, while lowspatial-frequency classical ripples are then formed as hills between individual expanding events [18].

Hence, we propose that the ripple formation observed in this study is consistent with the coupling of the incident electromagnetic radiation with plasmon charge oscillations. It can be proposed that the roughness of the initial surface is sufficient to initiate this plasmon polariton coupling. When overlapped pulses are used, the nanostructures/ripples created from preceding pulses, will also lead to such coupled surface excitations. The coupled plasmon polariton modes in turn decay non-radiatively and thereby create a unique spatial energy distribution across the surface. Using recently reported results [49] it can be speculated that this distribution leads to the bifuricated and bended ripple structure observed throughout Figs. 1, 2 and 3. 
The XRD patterns of the as-cast $\mathrm{Fe}_{51} \mathrm{Mn}_{10} \mathrm{Cr}_{4} \mathrm{Mo}_{12} \mathrm{C}_{15} \mathrm{~B}_{6} \mathrm{Er}_{2}$ alloy and the FSPLI treated $\left(3 \times 3 \mathrm{~mm}^{2}\right)$ sample are shown in Fig. 4 . The diffractogram of the as-cast sample consists of two broad halos with virtually no sharp peaks superimposed. This indicates that the as-cast rod is mainly amorphous. Conversely, clear diffraction peaks are observed in the XRD pattern of the pulsed laser treated sample. Both metallic and oxide phases are generated during FSPLI. Crystallization is expected because, in spite of the short duration of the pulses, temperature is often estimated to locally reach thousands of $\mathrm{K}$ in this type of processes [29,50], thus much higher than the glass transition, crystallization and even liquidus temperatures of the $\mathrm{Fe}_{51} \mathrm{Mn}_{10} \mathrm{Cr}_{4} \mathrm{Mo}_{12} \mathrm{C}_{15} \mathrm{~B}_{6} \mathrm{Er}_{2}$ alloy [46].

It is remarkable that some of the crystallized phases are ferromagnetic at room temperature ( $\alpha$-Fe and $\mathrm{Fe}_{3} \mathrm{C}$ exhibit Curie temperatures, $T_{\mathrm{C}}$, of $1044 \mathrm{~K}$ and $480 \mathrm{~K}$, respectively) [51], whereas $(\mathrm{Fe}, \mathrm{Mn})_{3} \mathrm{O}_{4}$ and $\mathrm{Fe}_{2} \mathrm{CrO}_{4}$ are both ferrimagnetic $\left(T_{\mathrm{C},(\mathrm{Fe}, \mathrm{Mn}) 3 \mathrm{O} 4}>570 \mathrm{~K}[52], T_{\mathrm{C}, \mathrm{Fe} 2 \mathrm{CrO} 4}=553 \mathrm{~K}\right.$ [53]). Other phases (e.g., $\mathrm{C}_{2} \mathrm{CrO}_{4}$ or $\mathrm{MoB}_{2}$ are neither ferromagnetic nor ferrimagnetic). In Fig. 5, EDX mappings reveal that the ridges are enriched in $\mathrm{Fe}, \mathrm{Mn}, \mathrm{Cr}$ and $\mathrm{O}$. Although the ripples structure is also observed in the $\mathrm{C}$ mapping, the zones enriched in $\mathrm{C}$ do not always exactly coincide with the ridges topology. In turn, Mo and Er are homogeneously distributed at the surface. These results indicate that the ferromagnetic/ferrimagnetic phases are mainly located at the ripples of the FSPLI regions. The deposited energy, delivered in this multi-pulse approach, appears sufficient to establish a temperature which enables redistribution of the atomic species. However, the precise mechanism for this effect is not clear, and hence it must be interpreted with caution.

Interestingly, the nanoripples can also be observed by AFM, not only when using the conventional topographic tapping mode [Fig. 6(a)] but particularly when the system is operated in the "Peak Force Quantitative Nanomechanical mode" (QNM mode) [Fig. 6 (b)]. For comparison, the SEM image of the same area is displayed in Fig. 6 (c). Under certain conditions, this QNM method allows extracting the mechanical properties of the scanned surface with sub-micrometer scale resolution. In peak-force tapping mode, the tip and the sample are intermittently brought into contact. However, the sample deformation depths are limited to a few nanometers and, therefore, only the elastic regime is actually probed. At such small applied force range, adhesion forces between the sample and the tip play an important role and they can be quantified. The 
adhesion mapping reveals maximum adhesion at the upper parts of the ripples and minimum contrast at the trenches. To our knowledge, this local variation of adhesion behavior after FSPLI has not been previously reported in the literature. Variations in adhesion of the order of hundreds of $\mathrm{nN}$ were observed between the different regions comprising the nanoripples [Fig. 6 (b)]. Interestingly, the average adhesion value in the FSPLI sample $\left(\left\langle F_{\text {adh }}\right\rangle \sim 190 \mathrm{nN}\right)$ is considerably higher than for the as-cast alloy (where the mean adhesion force is only around $30 \mathrm{nN}$ ), in agreement with previous results showing that an increase of surface roughness induces higher adhesion force in the material as a whole [42]. For soft materials (e.g., polymers) also the Young's modulus can be determined by AFM using the QNM operation mode [54]. However, for hard materials (like the one studied in this work), the quantification of Young's modulus using the QNM mode becomes rather unreliable. Thus, nanoindentation was employed to evaluate the Berkovich hardness, $H_{\mathrm{B}}$, and reduced Young's modulus $\left(E_{\mathrm{r}}\right)$ of the as-cast and laser treated samples. As shown in Fig. 6 (d), for a maximum load of $10 \mathrm{mN}$, higher penetration depth is attained in the laser treated sample, indicating that FSPLI induces mechanical softening at the surface. The hardness decreases from $H_{\mathrm{B}}=$ $14 \mathrm{GPa}$ to $H_{\mathrm{B}}=9.5 \mathrm{GPa}$ after FSPLI. Similarly, $E_{\mathrm{r}}$ decreases from 193 to $83 \mathrm{GPa}$. Such variations of surface mechanical properties can be ascribed to the increase of surface roughness caused by the laser irradiation $[55,56]$. The occurrence of roughness and surface corrugation is known to also play a crucial role in the hydrophobicity properties of surfaces. Here an increase of contact angle, from $64^{\circ}$ to $75^{\circ}$ is observed after FSPLI. This increase is quite modest compared to the results obtained from other rougher surfaces prepared by different methods. Namely, certain FSPLI conditions can render superhydrophobic surfaces by adjusting both the topological damage as well as the composition of the alloy at surface level [36].

Shown in Fig. 7 is the hysteresis loop of the $\mathrm{Fe}_{51} \mathrm{Mn}_{10} \mathrm{Cr}_{4} \mathrm{Mo}_{12} \mathrm{C}_{15} \mathrm{~B}_{6} \mathrm{Er}_{2}$ alloy after FSPLI surface treatment, recorded along the ripples direction. The clear ferromagnetic response is in contrast to the paramagnetic behavior of the as-cast alloy, as shown in the inset. Actually, more than one ferromagnetic-like contribution are visible in the hysteresis loop of the laser-treated sample, suggesting the co-existence of phases with lower coercivity (presumably due to the soft-magnetic $\alpha$-Fe and $\mathrm{Fe}_{3} \mathrm{C}$ phases) with others magnetically harder (such as the semi-hard ferrimagnetic $(\mathrm{Fe}, \mathrm{Mn})_{3} \mathrm{O}_{4}$ or $\mathrm{Fe}_{2} \mathrm{CrO}_{4}$ phases). Comparative AFM/MFM mappings of the classical ripples generated at the 
surface of the amorphous steel after FSPLI are shown in Fig. 8. The MFM images were acquired at the remanent state after applying a magnetic field $(H=5000 \mathrm{Oe})$ either parallel or perpendicular to the long axis of the ripples. In spite of the obvious analogies between the AFM (topological) and MFM (magnetic) images [cf. panels (a) and (c) with panels (b) and (d)], it is clear that the two types of mappings do not match each other, evidencing that the MFM contrast is not merely due to topological surface variations. Namely, dark and bright areas are observed inside each ripple in the MFM images, indicating a clear magnetic dipolar contrast. As expected, the orientation of such dipolar contrast depends on the direction of the previously applied magnetic field. Although the as-cast $\mathrm{Fe}_{51} \mathrm{Mn}_{10} \mathrm{Cr}_{4} \mathrm{Mo}_{12} \mathrm{C}_{15} \mathrm{~B}_{6} \mathrm{Er}_{2}$ alloy is paramagnetic, the magnetic contrast observed by MFM can be actually expected, given the ferro-/ferrimagnetic nature of the crystalline phases formed during the laser treatment at the alloy surface.

It should be noted that the magnetic patterning induced by FSPLI is accompanied with topological patterning and is irreversible in nature (i.e., it remains at the surface unless the sample is mechanically polished to remove the ripples). This is opposite to the ion-irradiation induced magnetic patterning in $\mathrm{Fe}_{60} \mathrm{Al}_{40}$ alloy [13], which preserved the smoothness of the surface and could be erased by subsequent low-temperature annealing treatments. In that case, however, a pre-lithographed mask was needed to define the local regions through which the ions could irradiate the atomically-ordered alloy, whereas FSPLI is completely mask-free. Hence, compared to ion-irradiation, FSPLI is a single-step magnetic patterning method and a cost-effective lithographic procedure. As aforementioned, nanoindentation can also generate arrays of ferromagnetic dots at the surface of various alloys, including metallic glasses. However, compared to FSPLI, nanoindentation is very slow. About one or two minutes are needed to make each indentation impression and induce the concomitant magnetic patterning. Hence, an array of $10 \times 10$ indentations covering an area of $1 \mathrm{~cm}^{2}$ would take about 2-3 hours. Conversely, inducing magnetic patterning in this $1 \mathrm{~cm}^{2}$ region by FSPLI needs only about 10 seconds. Thus, the FSPLI is much faster. Given the inherent topological patterning that accompanies FSPLI treatments, the induced magnetic structures could be used for applications like magnetic traps (of, for example, magnetic nanoparticles dispersed in fluids), magnetic sensors or magnetic encoders.

\section{Conclusion}


In summary, FSPLI has been shown to be a suitable method to generate periodic arrays of sub-micrometer-sized magnetic structures at the surface of non-ferromagnetic amorphous steel. The origin of the observed ferromagnetic behavior is the laser-induced devitrification of the glassy structure to form ferromagnetic $\left(\alpha-\mathrm{Fe}\right.$ and $\left.\mathrm{Fe}_{3} \mathrm{C}\right)$ and ferrimagnetic $\left[(\mathrm{Fe}, \mathrm{Mn})_{3} \mathrm{O}_{4}\right.$ and $\left.\mathrm{Fe}_{2} \mathrm{CrO}_{4}\right]$ phases. Besides the formation of ferromagnetic low-spatial-frequency ripples, much smaller nanoripples (of a few tens of $\mathrm{nm}$ in lateral size) are also generated during the FSPLI process, which is consistent with the coupling of the incident electromagnetic radiation with plasmon charge oscillations. Such nanoripples are clearly observed by high-resolution SEM and AFM and give rise to local variations of the adhesion force during AFM scanning. The observed variations in adhesion force can be particularly important for certain applications like traps for magnetic nanoparticles, where an exceedingly hydrophobic surface would not be desirable. The wettability and nanomechanical properties of the FSPLI have been also studied and compared to those of the as-cast amorphous alloy. The generation of magnetic structures by FSPLI turns out to be one of the fastest ways to induce magnetic patterning without the need of any shadow mask. The magnetic patterning procedure reported in this work can also be extrapolated to other materials, such as nonferromagnetic intermetallic alloys, austenitic steel or other types of non-magnetic metallic glasses, although the origin of the induced ferromagnetism could vary depending on the system under investigation.

\section{Acknowledgements}

This work has been partially funded by the 2014-SGR-1015 project from the Generalitat de Catalunya and the MAT2014-57960-C3-1-R (co-financed by the Fondo Europeo de Desarrollo Regional, FEDER) and MAT2014-57960-C3-2-R from the Spanish Ministerio de Economía y Competitividad (MINECO). Partial funding from the Consellería de Cultura, Xunta de Galicia, Spain, under the contract EM2012/019, is also acknowledged. Dr. Eva Pellicer is grateful to MINECO for the "Ramon y Cajal" contract (RYC-2012-10839).

\section{References}

[1] S.-C. Lee, A. A. Polycarpou, Adhesion Forces for Sub-10 nm Flying-Height Magnetic Storage Head Disk Interfaces, ASME J. Tribol. 126 (2004) 334-341. 
[2] V. Iacovacci, L. Ricotti, P. Dario, A. Menciassi, Design and Development of a Mechatronic System for Noninvasive Refilling of Implantable Artificial Pancreas, IEEE/ASME Trans. Mechatronics 20 (2014) 1160 -1169.

[3] M. Dkhil, A. Bolopion, S. Régnier, M. Gauthier, Optimization of the size of a magnetic microrobot for high throughput handling of micro-objects, IEEE/ASME Int. Conf. AIM (2014) 372-377.

[4] D. M. Drotlef, P. Blümler, A. del Campo, Magnetically Actuated Patterns for Bioinspired Reversible Adhesion (Dry and Wet), Adv. Mater. 26 (2014) 775-779.

[5] N. R. Khirad, R. K. Sanghi, D. J. Tidke, Magnetic Wall Climbing Devices - A Review, IOSR J. Mech. Civil Eng. 7 (2014) 55-59.

[6] G. Palasantzas, J. T. M. De Hosson, J. Barnas, Surface/interface roughness effects on magneto-electrical properties of thin films, Surf. Sci. 507-510 (2002) 541-545.

[7] Y. P. Zhao, R. M. Gamache, G. C. Wang, T. M. Lu, G. Palasantzas, J. T. M. De Hosson, Effect of surface roughness on magnetic domain wall thickness, domain size, and coercivity, J. Appl. Phys. 89 (2001) 1325-1330.

[8] J. I. Martín, J. Nogués, K. Liu, J. L. Vicent, I. K. Schuller, Ordered magnetic nanostructures: fabrication and properties, J. Magn. Magn. Mater. 256 (2003) 449-501.

[9] J. Sort, A. Concustell, E. Menéndez, S. Suriñach, M. D. Baró, J. Farran, J. Nogués, Selective generation of local ferromagnetism in austenitic stainless steel using nanoindentation, Appl. Phys. Lett. 89 (2006) 032509.

[10] J. Sort, A. Concustell, E. Menéndez, S. Suriñach, K. V. Rao, S. C. Deevi, M. D. Baró, J. Nogués, Periodic arrays of micrometer and sub-micrometer magnetic structures prepared by nanoindentation of a nonmagnetic intermetallic compound, Adv. Mater. 18 (2006) 1717-1720.

[11] J. Sort, L. F. Bonavina, A. Varea, C. Souza, W. J. Botta, C. S. Kiminami, C. Bolfarini, S. Suriñach, M. D. Baró, J. Nogués, Out-of-plane magnetic patterning based on indentation-induced nanocrystallization of a metallic glass, Small 6 (2010) 15431549.

[12] R. Bali, S. Wintz, F. Meutzner, R. Hübner, R. Boucher, A. A. Ünal, S. Valencia, A. Neudert, K. Potzger, J. Bauch, F. Kronast, S. Facsko, J. Lindner, J. Fassbender, Printing 
nearly-discrete magnetic patterns using chemical disorder induced ferromagnetism, Nano Lett. 14 (2014) 435-441.

[13] E. Menéndez, M. O. Liedke, J. Fassbender, T. Gemming, A. Weber, L. J. Heyderman, K. V. Rao, S. C. Deevi, S. Suriñach, M. D. Baró, J. Sort, J. Nogués, Direct magnetic patterning due to the generation of ferromagnetism by selective ion irradiation of paramagnetic FeAl alloys, Small 5 (2009) 229-234.

[14] C. De Marco, S. M. Eaton, R. Suriano, S. Turri, M. Levi, R. Ramponi, G. Cerullo, R. Osellame, Surface properties of femtosecond laser ablated PMMA, ACS Appl. Mater. Interfaces 2 (2010) 2377-2384.

[15] J. Jia, M. Li, C. V. Thompson, Amorphization of silicon by femtosecond laser pulses, Appl. Phys. Lett. 84 (2004) 3205-3207.

[16] L. L. Ran, S. L. Qu, Structure formation on the surface of alloys irradiated by femtosecond laser pulses, Appl. Surf. Sci. 256 (2010) 2315-2318.

[17] N. Yasumaru, E. Sentoku, K. Miyazaki, J. Kiuchi, Femtosecond-laser-induced nanostructure formed on nitrided stainless steel, Appl. Surf. Sci. 264 (2013) 611-615.

[18] J. Vincenc Obona, V. Ocelík, J. Z. P. Z. P. Skolski, V. S. S. Mitko, G. R. B. E. Römer, A. J. J. Huis in't Veld, J. T. M. De Hosson, On the surface topography of ultrashort laser pulse treated steel surfaces, Appl. Surf. Sci. 258 (2011) 1555-1560.

[19] J. Z. P. Skolski, G. R. B. E. Römer, J. V. Obona, V. Ocelik, A. J. Huis In’t Veld, J. T. M. De Hosson, Laser-induced periodic surface structures: Fingerprints of light localization, Phys. Rev. B - Condens. Matter Mater. Phys. 85 (2012) 1-9.

[20] W. Zhang, G. H. Cheng, Q. Feng, L. M. Cao, Picosecond laser-induced formation of spikes in a single crystal superalloy, Appl. Surf. Sci. 258 (2012) 9452-9456.

[21] W. Zhang, G. Cheng, X. D. Hui, Q. Feng, Abnormal ripple patterns with enhanced regularity and continuity in a bulk metallic glass induced by femtosecond laser irradiation, Appl. Phys. A 115 (2014) 1451-1455.

[22] F. X. Ma, J. J. Yang, X. N. Zhu, C. Y. Liang, H. S. Wang, Femtosecond laserinduced concentric ring microstructures on Zr-based metallic glass, Appl. Surf. Sci. 256 (2010) 3653-3660.

[23] A. E. Siegman, P. M. Fauchet, Stimulated Wood's anomalies on laser-illuminated surfaces, IEEE J. Quantum Electron. 22 (1986) 1384-1403.

[24] M. Huang, F.L. Zhao, Y. Cheng, N.S. Xu, Z.Z. Xu, Origin of Laser-Induced NearSubwavelength Ripples: Interference between Surface Plasmons and Incident Laser, ACS Nano, 3 (2009) 4062-4070. 
[25] T. J. Y. Derrien, R. Koter, J. Crüger, S. Höhm, A. Rosenfeld, J. Bonse, Plasmonic formation mechanism of periodic 100-nm-structures upon femtosecond laser irradiation of silicon in water, J. Appl. Phys. 116 (2014) 074902.

[26] J. C. Wang, C. L. Guo, Ultrafast dynamics of femtosecond laser-induced periodic surface pattern formation on metals, Appl. Phys. Lett. 87 (2005) 251914.

[27] F. Garrelie, J. Colombier, F. Pigeon, S. Tonchev, N. Faure, M. Bounhalli, S. Reynaud, and O. Parriaux, Evidence of surface plasmon resonance in ultrafast laserinduced ripples, Opt. Express 19, 9035-9043 (2011)

[28] J. Reif, O. Varlamova, F. Costache, Femtosecond laser induced nanostructure formation: self-organization control parameters, Appl. Phys. A - Mater. Sci. Process., 92 (2008) 1019-1024.

[29] L. V. Zhigilei, Z. B. Lin, D. S. Ivanov, Atomistic modeling of short pulse laser ablation of metals: connections between melting, spallation, and phase explosion, J. Phys. Chem. C 113 (2009) 11892-11906.

[30] E. Bévillon, J. P. Colombier, B. Dutta, R. Stoian, Ab initio nonequilibrium thermodynamic and transport properties of ultrafast laser irradiated 316L stainless steel, J. Phys. Chem. C 119 (2015) 11438-11446.

[31] Y. Zhang, L. Liu, G. Zou, N. Chen, A. Wu, H. Bai, Y. Zhou, Femtosecond laserinduced phase transformations in amorphous $\mathrm{Cu}_{77} \mathrm{Ni}_{6} \mathrm{Sn}_{10} \mathrm{P}_{7}$ alloy, J. Appl. Phys. 117 (2015) 023109.

[32] W. Zhang, G. H. Cheng, Q. Feng, L. M. Cao, F. P. Wang, R. Q. Hui, Abrupt transition from wavelength structure to subwavelength structure in a single-crystal superalloy induced by femtosecond laser, Appl. Surf. Sci. 257 (2011) 4321-4324.

[33] G. J. Zhang, D. H. Gu, F. X. Gan, X. W. Jiang, Q. X. Chen, Femtosecond laserinduced crystallization in amorphous $\mathrm{Ge}_{2} \mathrm{Sb}_{2} \mathrm{Te}_{5}$ films, Thin Solid Films 474 (2005) 169-172.

[34] B. Q. Chen, Y. Li, Y. Cai, R. Li, S. J. Pang, T. Zhang, Surface vitrification of alloys by laser surface treatment, J. Alloys Compd. 511 (2012) 215-220.

[35] S. Valette, P. Steyer, L. Richard, B. Forest, C. Donnet, E. Audouard, Influence of femtosecond laser marking on the corrosion resistance of stainless steels, Appl. Surf. Sci. 252 (2006) 4696-4701.

[36] A. M. Kietzig, S. G. Hatzikiriakos, P. Englezos, Patterned superhydrophobic metallic surfaces, Langmuir 25 (2009) 4821-4827. 
[37] C. C. Yu, J. P. Chu, C. M. Lee, W. Diyatmika, M. H. Chang, J. Y. Jeng, Y. Yokoyama, Bending property enhancements of $\mathrm{Zr}_{55} \mathrm{Cu}_{30} \mathrm{Al}_{10} \mathrm{Ni}_{5}$ bulk metallic glass: effects of various surface modifications, Mater. Sci. Eng. A 633 (2015) 69-75.

[38] J. Bonse, J. Krüger, Pulse number dependence of laser-induced periodic surface structures for femtosecond laser irradiation of silicon, J. Appl. Phys. 108 (2010) 034903. [39] C. Smith, S.. Katakam, S.. Nag, X.. Chen, R.V.. Ramanujan, N.B.. Dahotre, et al., Improved soft magnetic properties by laser de-vitrification of $\mathrm{Fe}-\mathrm{Si}-\mathrm{B}$ amorphous magnetic alloys, Mater. Lett. 122 (2014) 155-158.

[40] J. Z. P. Skolski, G. R. B. E. Römer, J. V Obona, V. Ocelik, A. J. Huis, J. T. M. De Hosson, Inhomogeneous Absorption of Laser Radiation:Trigger of LIPSS Formation, J. Laser Micro Nanoeng. 8 (2013) 1-5.

[41] S. Vilain, J. Ebothe, M. Troyon, Surface roughness and composition effects on the magnetic properties of electrodeposited Ni-Co alloys, J. Magn. Magn. Mater. 157-158 (1996) 274-275.

[42] G. Palasantzas, J. T. M. De Hosson, Influence of surface roughness on the adhesion of elastic films, Phys. Rev. E. Stat. Nonlin. Soft Matter Phys. 67 (2003) 021604.

[43] Z. P. Lu, C.T. Liu, J. R. Thompson, W. D. Porter, Structural amorphous steels, Phys. Rev. Lett. 92 (2004) 245503.

[44] M. J. Duarte, J. Klemm, S. O. Klemm, K. J. J. Mayrhofer, M. Stratmann, S. Borodin, A. H. Romero, M. Madinehei, D. Crespo, J. Serrano, S. S. A. Gerstl, P. P. Choi, D. Raabe, F. U. Renner, Element-resolved corrosion analysis of stainless-type glass-forming steels, Science 341 (2013) 372-376.

[45] V. Ponnambalam, S. J. Poon, G. J. Shiflet, V. M. Keppens, R. Taylor, G. Petculescu, Synthesis of iron-based bulk metallic glasses as nonferromagnetic amorphous steel alloys, Appl. Phys. Lett. 83 (2003) 1131-1133.

[46] V. Ponnambalam, S. J. Poon, G. J. Shiflet, Fe-Mn-Cr-Mo-(Y,Ln)-C-B (Ln = Lanthanides) bulk metallic glasses as formable amorphous steel alloys, J. Mater. Res. 19 (2004) 3046-3052.

[47] W. C. Oliver, G. M. Pharr, An improved technique for determining hardness and elastic modulus using load and displacement sensing indentation experiments, J. Mater. Res. 7 (1992) 1564-1583.

[48] T. Ahn, S. Oh, X. Hu, J. W. Lee, C. W. Park, H. M. Yang, C. Kim, J. D. Kim, Controlled self-assembly for high-resolution magnetic printing, Small 10 (2014) 10811085 . 
[49] S. He, J. J. J. Nivas, K. K. Anoop, A. Vecchione, M. Hu, R. Bruzzese, S. Amoruso, Surface structures induced by ultrashort laser pulses: Formation mechanisms of ripples and grooves, Appl. Surf. Sci. 353 (2015) 1214-1222.

[50] S. M. Eaton, H. Zhang, P. R. Herman, F. Yoshino, L. Shah, J. Bovatsek, A. Y. Arai, Heat accumulation effects in femtosecond laser-written waveguides with variable repetition rate, Opt. Express 13 (2005) 4708-4716.

[51] M. Eisenbach, D. M. Nicholson, A. Rusanu, G. Brown, First principles calculation of finite temperature magnetism in $\mathrm{Fe}$ and $\mathrm{Fe}_{3} \mathrm{C}$, J. Appl. Phys. 109 (2011) 07E138.

[52] V. A. M. Brabers, Comment on "Size-dependent Curie temperature in nanoscale $\mathrm{MnFe}_{2} \mathrm{O}_{4}$ particles”, Phys. Rev. Lett. 68 (1992) 3113.

[53] V. I. Maksimochkin, R. R. Gubaidullin, M. Ya. Gareeva, Magnetic properties and structure of $\mathrm{Fe}_{2-x} \mathrm{Mg}_{x} \mathrm{CrO}_{4}$ chromites, Moscow Univ. Phys. Bull. 68 (2013) 241-248.

[54] J. Adamcik, A. Berquand, R. Mezzenga, Single-step direct measurement of amyloid fibrils stiffness by peak force quantitative nanomechanical atomic force microscopy, Appl. Phys. Lett. 98 (2011) 193701.

[55] C. Walter, C. Mitterer, 3D versus 2D finite element simulation of the effect of surface roughness on nanoindentation of hard coatings, Surf. Coat. Technol. 203 (2009) 3286-3290.

[56] J. Y. Kim, S. K. Kang, J. J. Lee, J. I. Jang, Y. H. Lee, D. Kwon, Influence of surface-roughness on indentation size effect, Acta Mater. 55 (2007) 3555-3562. 


\section{Figure captions}

Fig. 1. (a) SEM images (secondary electrons) of different patterns written at the surface of the $\mathrm{Fe}_{51} \mathrm{Mn}_{10} \mathrm{Cr}_{4} \mathrm{Mo}_{12} \mathrm{C}_{15} \mathrm{~B}_{6} \mathrm{Er}_{2}$ alloy by femtosecond pulsed laser irradiation (FSPLI) with $95 \%$ overlapped 500 fs laser pulses and applied fluence $0.33 \mathrm{~J} / \mathrm{cm}^{2}$; (b) detail of a circular dot obtained by FSPLI imaged by SEM; (c) magnified SEM view of the socalled "classical ripples" formed during the FSPLI process, whose direction is perpendicular to the polarization of the incident laser $(P)$.

Fig. 2. (a) AFM image of the classical ripples; (b) representative AFM line profile measured along the direction orthogonal to the ripples long axis.

Fig. 3. The magnified views of the classical ripples at the FSPLI treated surfaces (a) without and (b) with vertically tilting the sample plane by $45^{\circ}$ with respect to the $x-y$ plane separately, showing the presence and the depth of "nanoripples" at the trenches between consecutive ridges, with orientation parallel to the polarization direction of the incident laser beam $(\mathrm{P})$.

Fig. 4. X-ray diffraction patterns corresponding to the as-cast glassy alloy and the FSPLI surface-treated alloy.

Fig. 5. Secondary-electrons SEM image of the classical ripples, together with the corresponding elemental mappings (Fe, Mn, $\mathrm{Cr}, \mathrm{Mo}, \mathrm{C}, \mathrm{Er}$ and $\mathrm{O}$ ), as obtained from energy-dispersive X-ray (EDX) analyses.

Fig. 6. Images of FSPLI treated circle pattern in Fig. 1(a): (a) topography and (b) adhesion force of the FSPLI treated surface, both obtained by AFM. For comparison, (c) is an SEM image of the same area as the AFM images. (d) Representative load $(F)$ displacement $(h)$ nanoindentation curves corresponding to the as-cast and FSPLI treated samples.

Fig. 7. Hysteresis loop corresponding to the $\mathrm{Fe}_{51} \mathrm{Mn}_{10} \mathrm{Cr}_{4} \mathrm{Mo}_{12} \mathrm{C}_{15} \mathrm{~B}_{6} \mathrm{Er}_{2}$ alloy after FSPLI treatment, applying the magnetic field parallel to the ripples direction. The inset shows the dependence of the magnetization $(M)$ as a function of applied magnetic field $(H)$ for the as-cast $\mathrm{Fe}_{51} \mathrm{Mn}_{10} \mathrm{Cr}_{4} \mathrm{Mo}_{12} \mathrm{C}_{15} \mathrm{~B}_{6} \mathrm{Er}_{2}$ alloy, which is paramagnetic. 
Fig. 8. (a,c) representative AFM images of the classical ripples together with $(b, d)$ the corresponding MFM images obtained at remanence after having saturated the sample with a strong magnetic field $(H=5000 \mathrm{Oe})$ along the directions perpendicular and parallel to the ripples long axis (as indicated by the white arrows), respectively. 
Figure 1
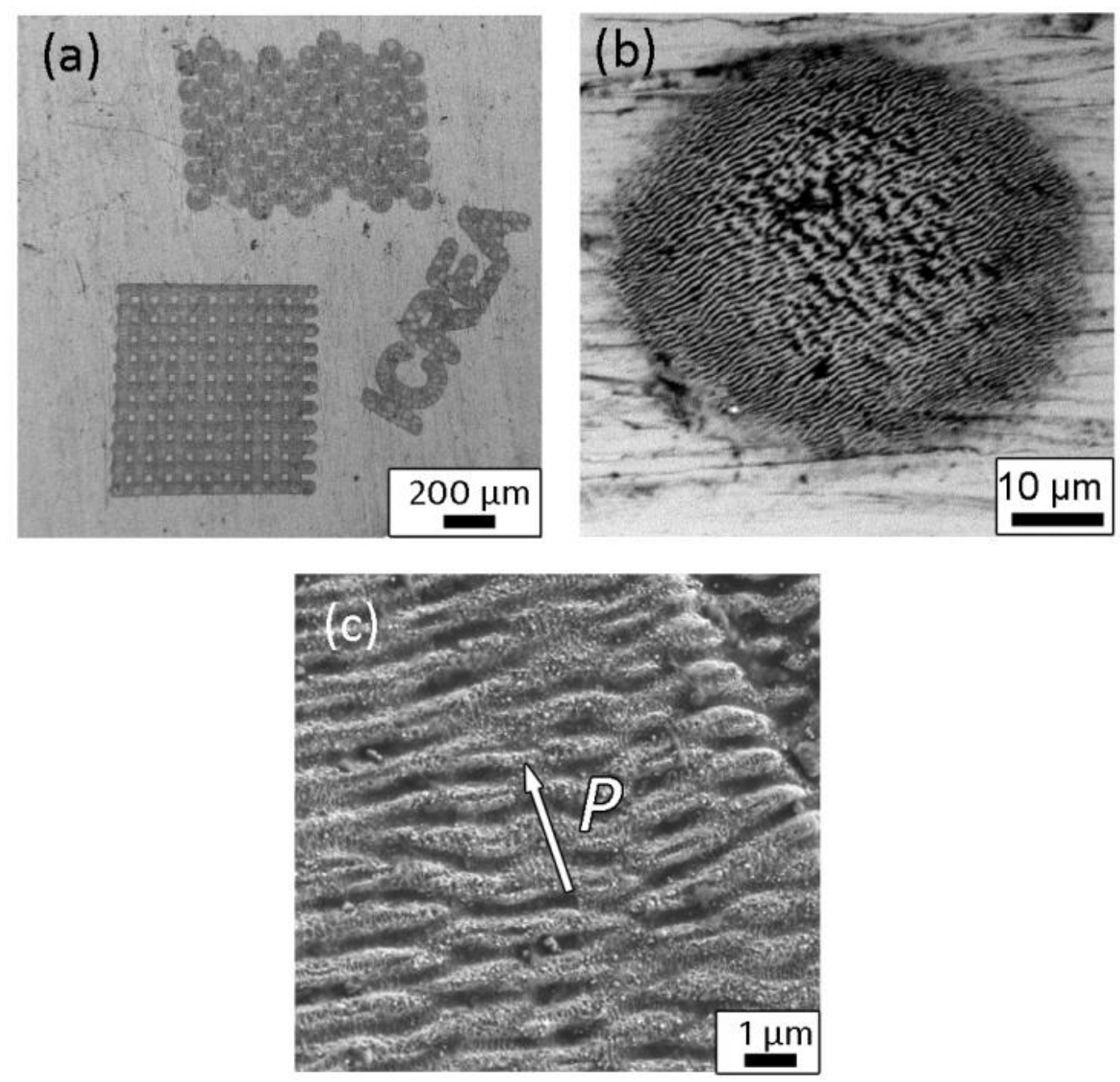
Figure 2
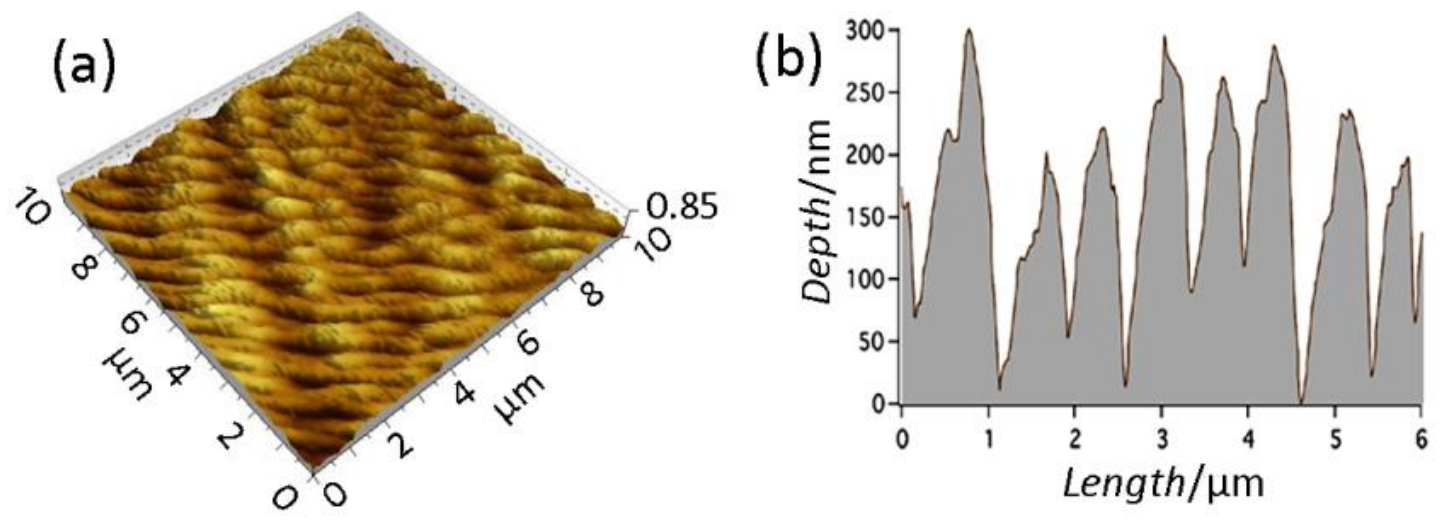
Figure 3
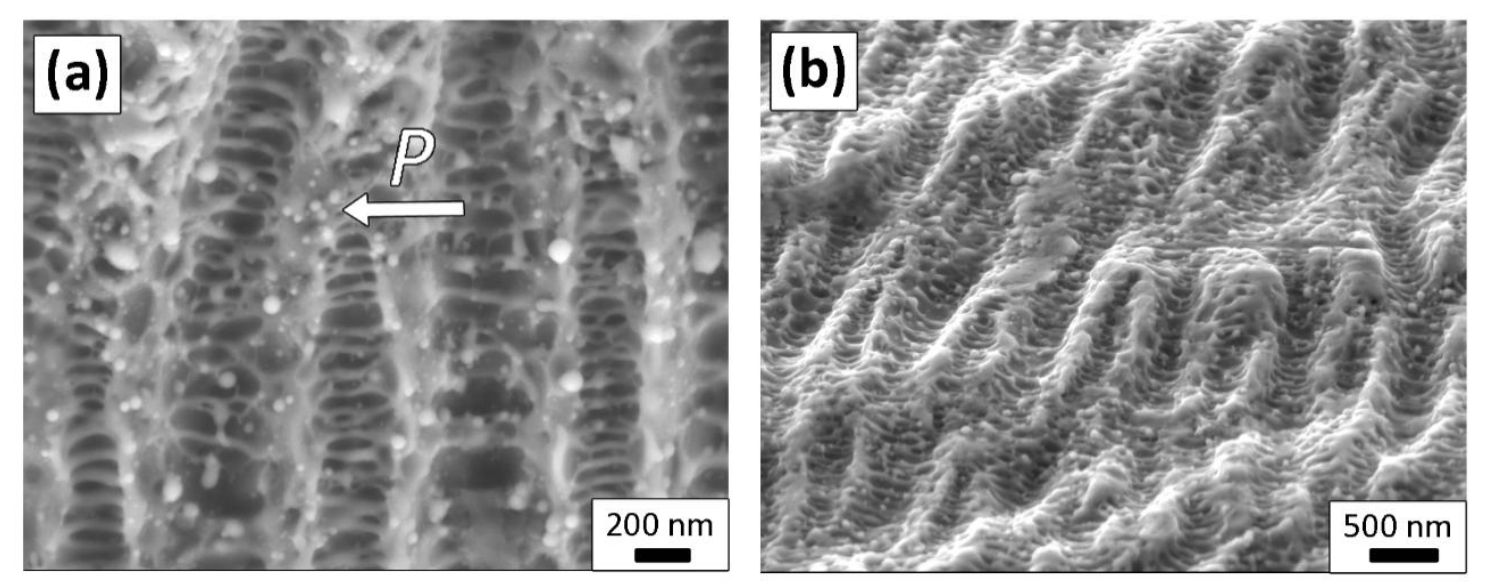
Figure 4

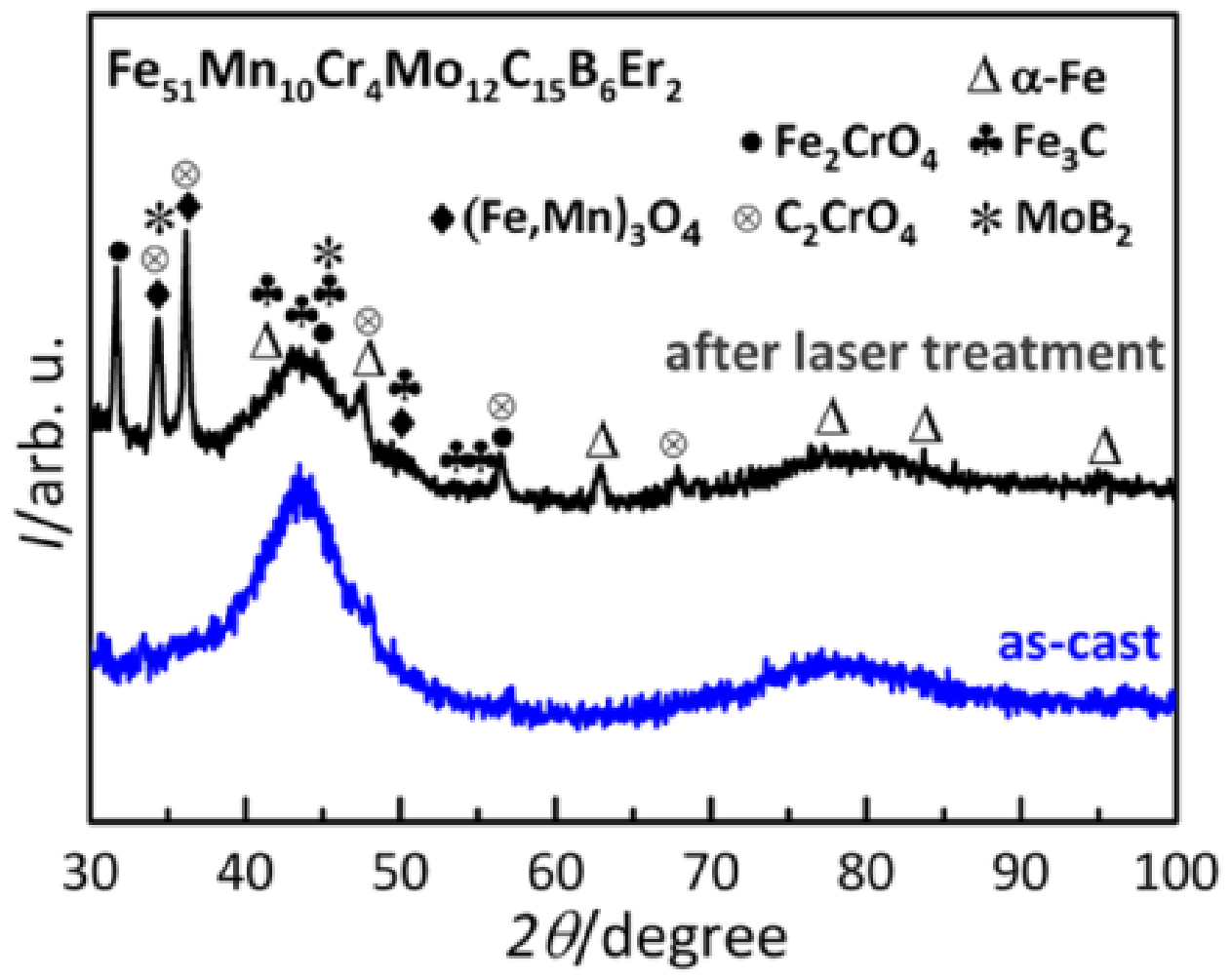




\section{Figure 5}
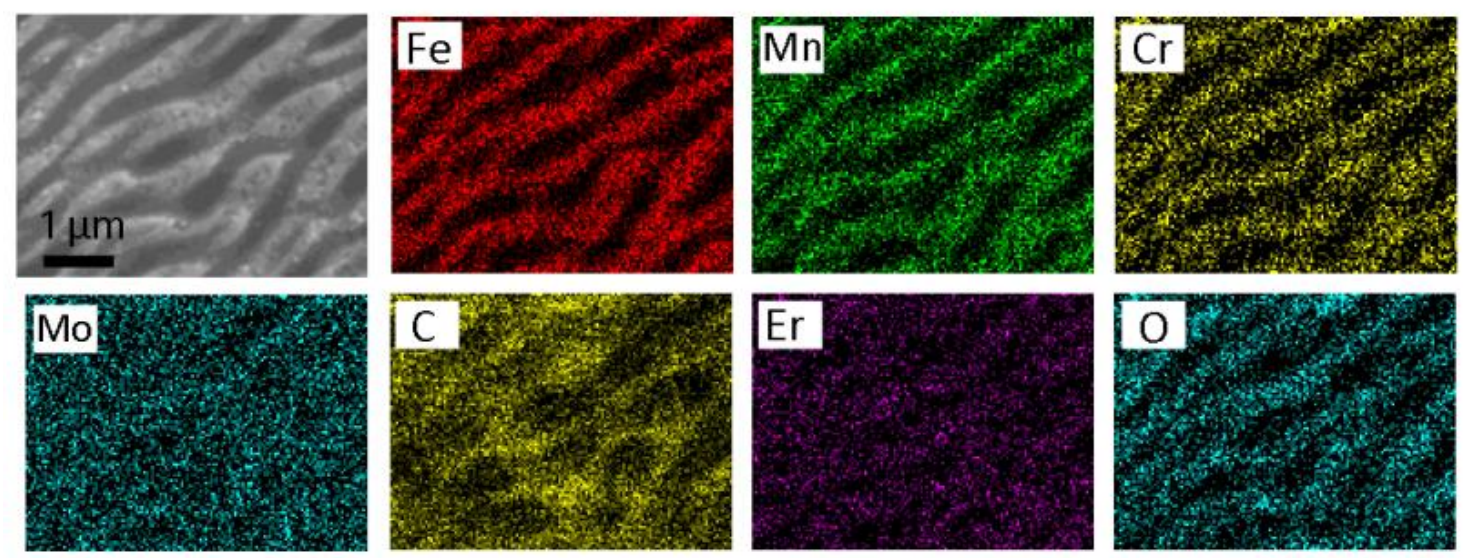
Figure 6
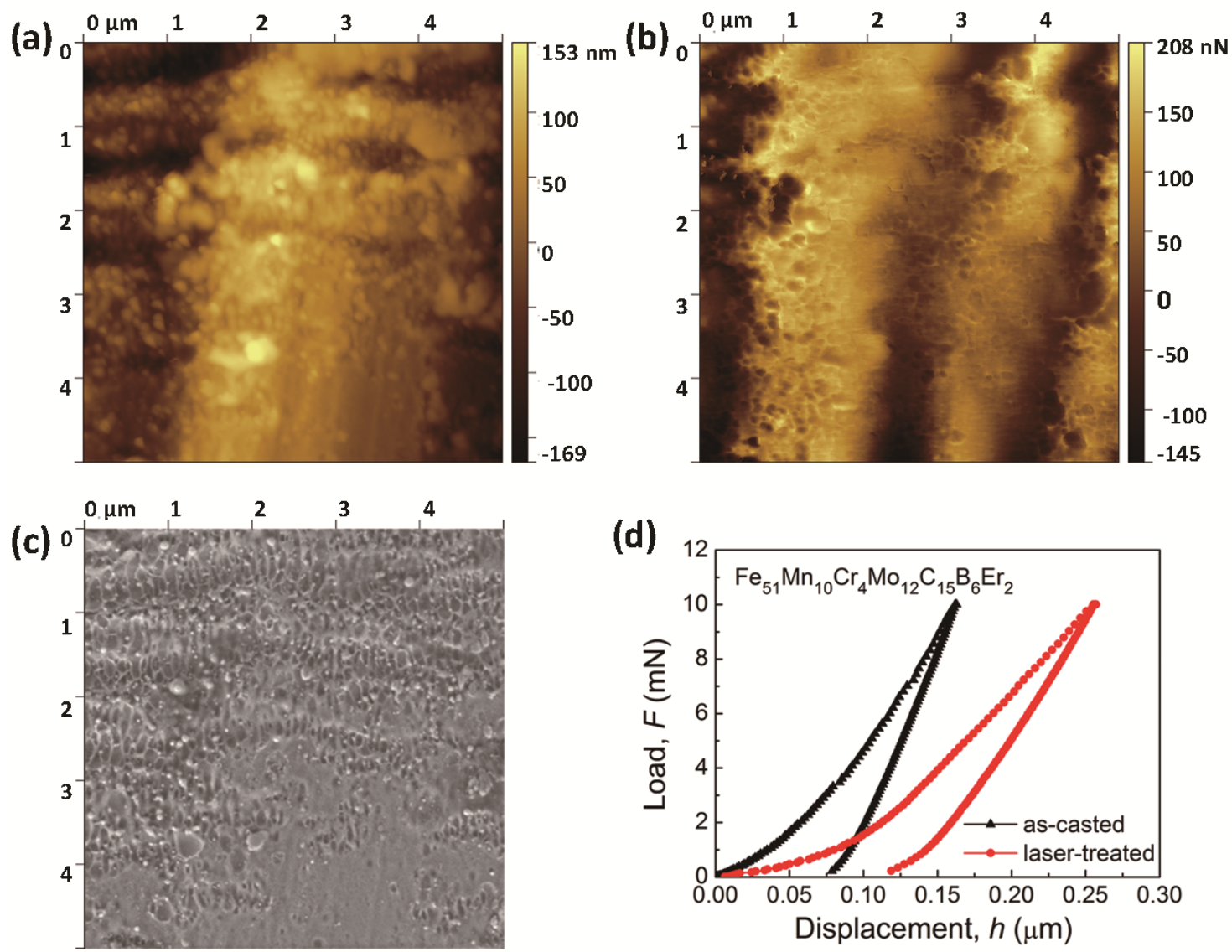

(d)

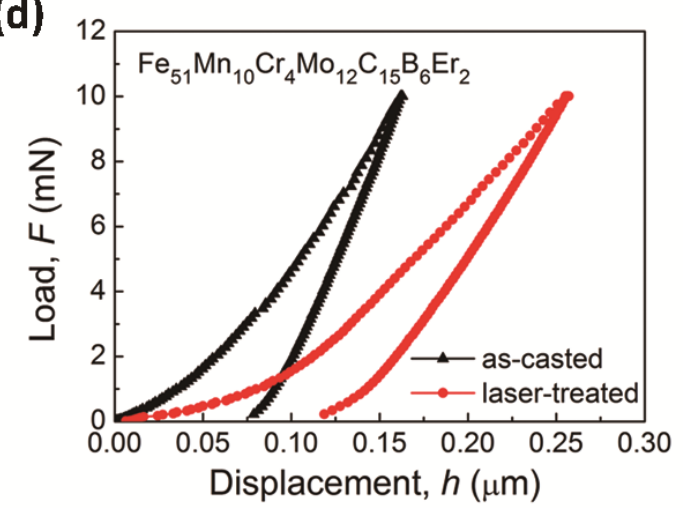


Figure 7

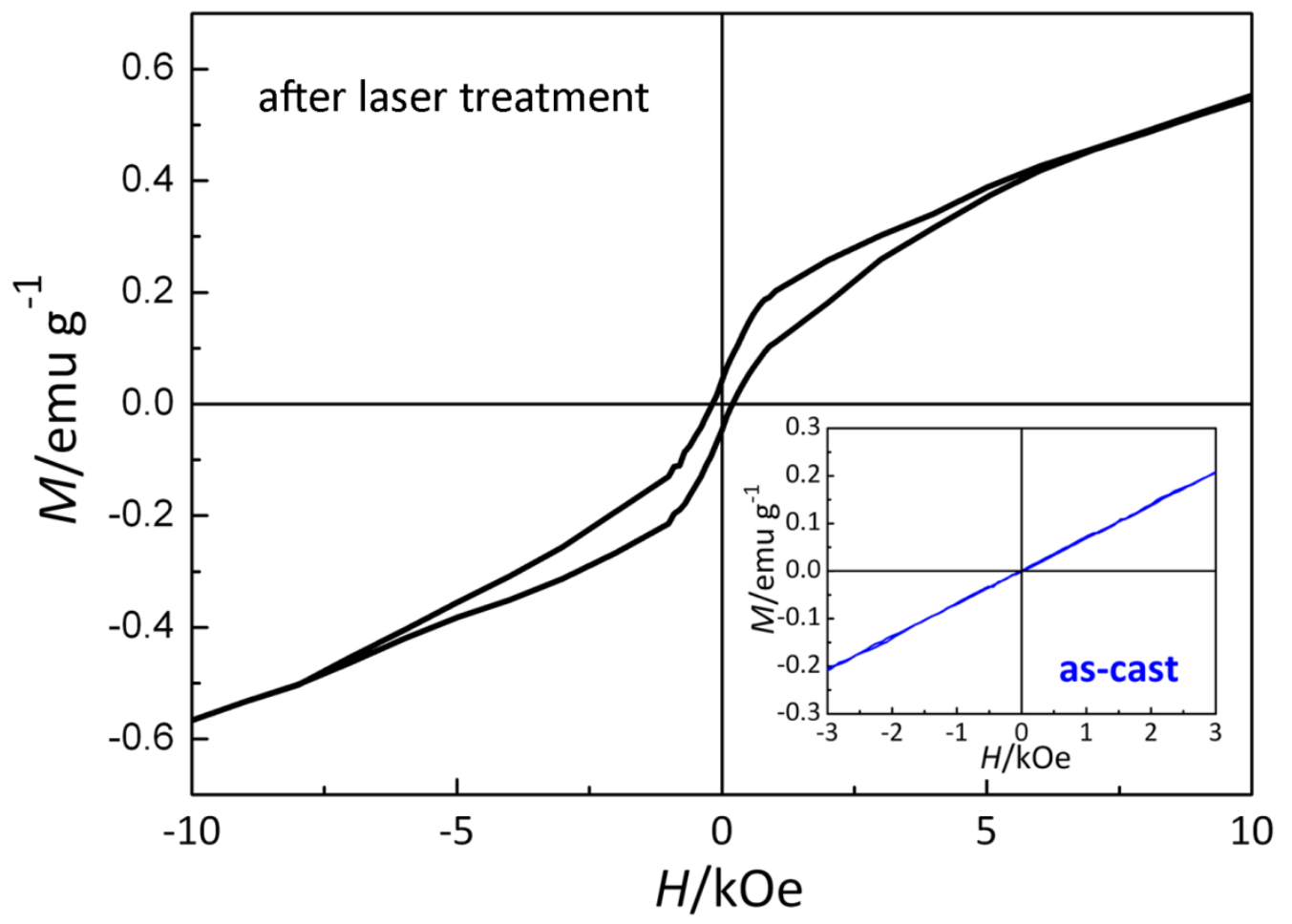


Figure 8

(a)

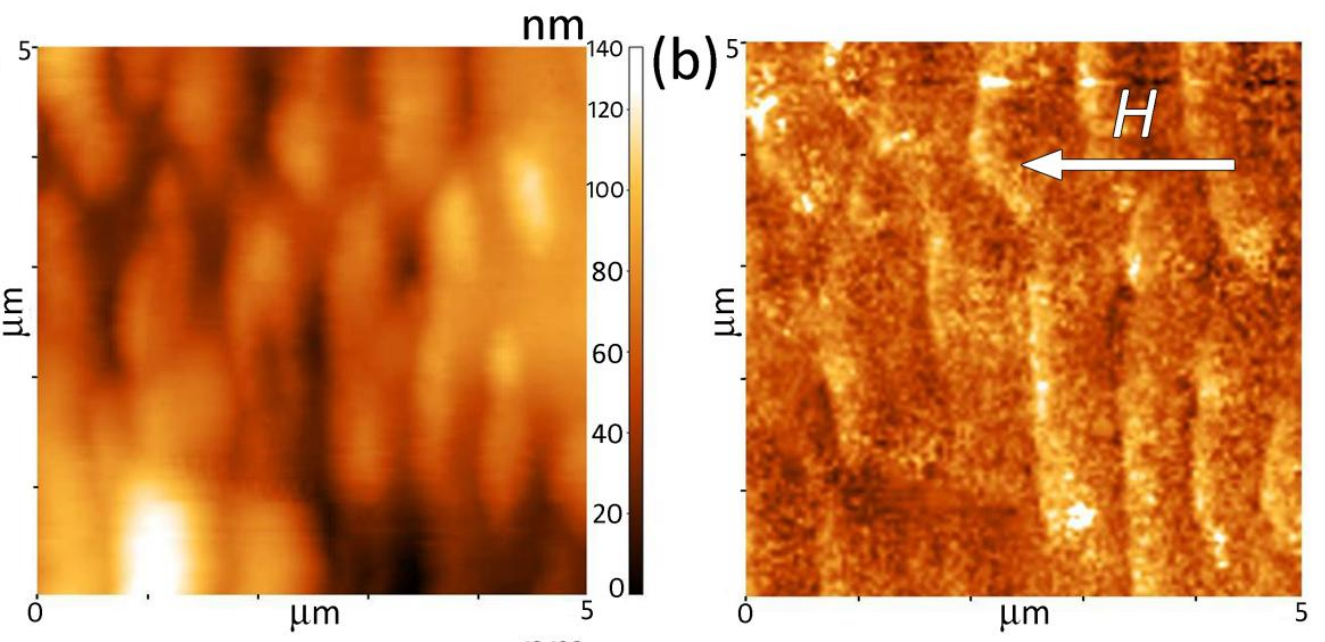

(c)

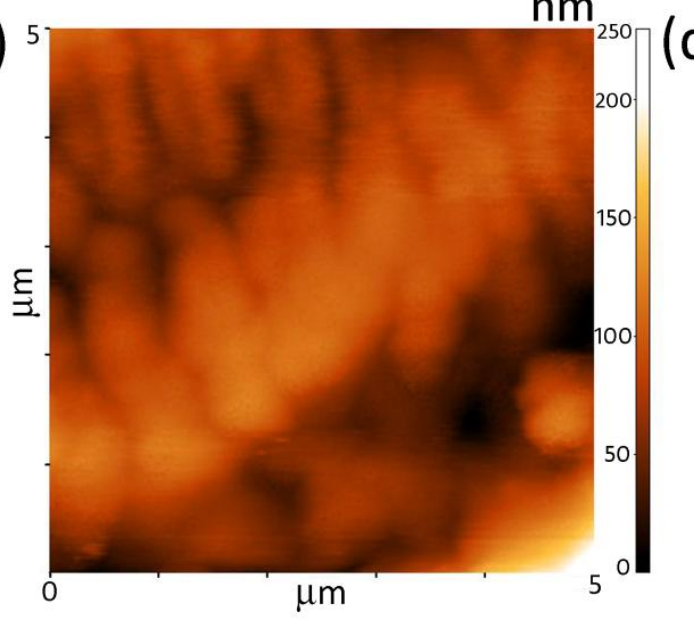

(d)

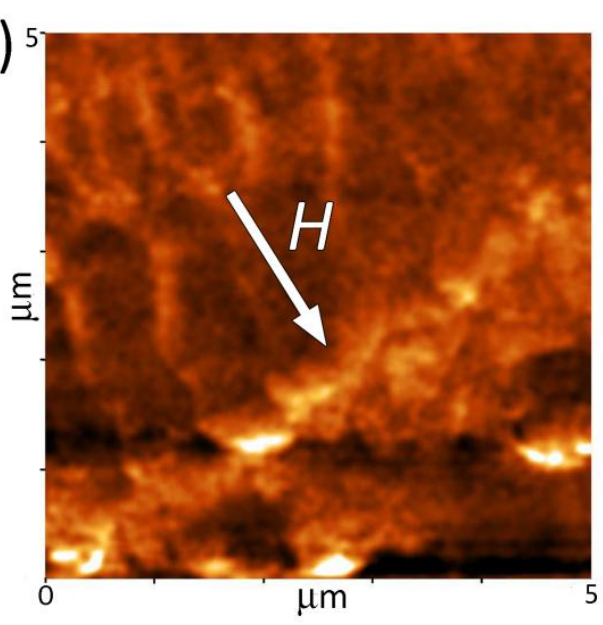

